\title{
BMJ Open Preferences for everyday living inventory (PELI): study protocol for piloting a culture-sensitive and setting- specific translated instrument in German care settings (PELI-D)
}

Tobias Ingo Stacke (1) ,1,2 Johannes Michael Bergmann, ${ }^{1,2}$ Armin Michael Ströbel, ${ }^{1,2}$ René Müller-Widmer, ${ }^{1}$ Daniel Purwins, ${ }^{1,2}$ Christina Manietta, ${ }^{1,2}$ Mike Rommerskirch, ${ }^{1,2}$ Anna-Eva Nebowsky, ${ }^{1,2}$ Anne Wegner, ${ }^{3}$ Jane White, ${ }^{4}$ Heidemarie Kelleter, ${ }^{5}$ Nada Ralic, ${ }^{6}$ Kimberly Van Haitsma, ${ }^{7}$ Martina Roes ${ }^{1,2}$

To cite: Stacke $\mathrm{Tl}$,

Bergmann JM, Ströbel AM, et al. Preferences for everyday living inventory (PELI): study protocol for piloting a culture-sensitive and setting-specific translated instrument in German care settings (PELI-D). BMJ Open 2020;10:e030268. doi:10.1136/ bmjopen-2019-030268

- Prepublication history for this paper is available online. To view these files, please visit the journal online (http://dx.doi. org/10.1136/bmjopen-2019030268).

Received 12 March 2019 Revised 12 November 2019 Accepted 20 November 2019

Check for updates

(C) Author(s) (or their employer(s)) 2020. Re-use permitted under CC BY-NC. No commercial re-use. See rights and permissions. Published by BMJ.

For numbered affiliations see end of article.

Correspondence to

Tobias Ingo Stacke;

tobias-ingo.stacke@dzne.de

\section{ABSTRACT}

Introduction Regardless of the healthcare setting, person-centred care and its implementation in caring for older people are a central issue for those who are responsible as professional caregivers and for those in need of care within the care process. Both aspects encompass the possibility of recognising personal preferences. To provide person-centred care, professional caregivers need to know about the individual preferences of the persons being cared for. Therefore, the PELI (an acronym for 'Preferences for Everyday Living Inventory') instrument was developed at the Polisher Research Institute (USA) for the systematic recording of individual preferences of older people in need of care. There is currently no comparable instrument available in the German language.

Methods As part of the proposed project PELI-D, all versions of the original PELI instrument (nursing home version) were (1) culture-sensitively translated into German and will be (2) examined in a pilot study for their reliability, feasibility and practicability. For the project PELI-D, we worked together with our practice partners in Germany (Diaconia and Caritas in North RhineWestphalia) and collaborated with our partners in the USA who developed the PELI instrument. This study protocol focuses on the pilot study, which will be conducted by the German Center for Neurodegenerative Diseases (DZNE) (site Witten).

Ethics and dissemination This study was approved by the internal quality control committee of the DZNE (ID number: WI029 PELI-D) and by the ethics committee of the German Society of Nursing Science Duisburg branch office (ID number: 18-010). All personal information will be deidentified with a specific identification code and stored in a secured location apart from the rest of the study data. Only qualified and study-related staff will be allowed access to the data. The results of the study will be distributed nationally and internationally through peerreviewed journals, conferences and journals for nursing care practice.
Strengths and limitations of this study

- The culture-sensitive translation is comprehensive and strengthens the understanding of the preferences for everyday living inventory (PELI-D) questionnaire within the specific care settings.

- The PELI-D instrument and the contained general preferences will be discussed in different care settings, applying qualitative and quantitative methods.

- Regular meetings were established with the PELI developers to reflect on the approach and discuss preliminary results.

- Influencing factors can affect the response behaviour of the people in need of care with regard to their preferences. Such factors include, for example, the social context (individual and regional), the specific processes and structures in which care is provided, and the Christian-centred self-conception of our cooperation partners. With regard to our convenience sample, we could only consider a specific perspective older people in need of care.

\section{INTRODUCTION}

A self-determined life and social participation for older people in need of care are essential political goals for care services. In Germany, those goals can be found in the demographicstrategic considerations of the federal government, ${ }^{1}$ in the National Action Plan for the Implementation of the UN Convention on the Rights of Persons with Disabilities, ${ }^{2}$ in the federal law on participation ${ }^{3}$ and in the Expert Standard to foster and sustain relationships for people with dementia. ${ }^{4}$

Knowledge of the everyday life preferences of older people in need of care is the foundation on which ongoing individualised care planning is based. Therefore, an instrument 
for recording preferences in the area of everyday life seemed to be helpful to emphasise the important policy objective of achieving person-centeredness. In this context, the PELI (acronym for 'Preferences for Everyday Living Inventory') instrument was developed at the Polisher Research Institute (US) for the systematic recording of individual preferences of older people in need of care. ${ }^{56}$

The PELI instrument (current version for nursing homes) contains 72 main items, all of which include a rating of how important a certain preference is for the person being interviewed. ${ }^{7}$ There are questions such as 'How important is it to you [... to choose what name you would like me to use when I greet you?], [... to choose how to care for your mouth?], [... to be involved in cooking?]. 'The importance of each PELI item is ranked on a 4-point Likert scale. If a preference appears "important" or "important but can't do (it anymore)", more detailed questions will be asked in a semistandardised way. The data collection can be conducted as interviews with older people in need of care by professional caregivers (selfversion) or independently of older people in need of care as an interview with their close relatives (proxy-version). ${ }^{58}$

The PELI was developed based on a comprehensive theoretical framework, ${ }^{9}$ bolstered by cognitive interviewing techniques ${ }^{8}$ that ensured that wording took into account ideographic older adult care recipient perspectives. Since July 2016, the PELI-nursing home version has been tested in two states in the USA. Previous study results in the USA have shown that the PELI instrument is a feasible, meaningful and valid instrument. ${ }^{57810}$

In a preliminary study at the German Center for Neurodegenerative Diseases (DZNE site Witten) in 2017, a self-developed questionnaire was used to explore if and how preferences of older people in need of care are described in the nursing record. ${ }^{11}$ For this research, professional caregivers were asked to read out loud the preferences that were written in the nursing record for anonymous selected older people in need of care. One of the key results was that, although there are only a few preferences listed in the nursing record, they were inadequately covered in terms of content or consistency. This was a major reason to do further research on this topic. The PELI, as described above, is an instrument-which represents the perspective of older adult care recipientsthat enables to trace personal preferences of people in need of care in a structured way and therefore, actively supports person-centred care.

After further search we concluded that currently, to our knowledge, there is no other idiosyncratic, itembased instrument in German comparable to the PELI. To fill this gap, the DZNE (site Witten) first developed a cultural-sensitive translation and item-based modification of the original PELI questionnaire to German for three specific care settings: nursing homes, home care and adult daycares (2017-2018). Second, the specific translated and modified PELI questionnaires (PELI-D) will be applied in the specific settings by trained professional caregivers, tested with psychometrical approaches and evaluated by qualitative interviews (2018-2019). This step is critical to provide more information about care provider perspectives on fulfilling the preferences of older people receiving care in their settings. The main goal is to make the PELI-D questionnaire available to different German care settings free of charge. This will be done in close cooperation with the practice partners in Germany (Diocesan Caritas Association for the Archdiocese of Cologne (registered association) and Diaconia Dusseldorf) and with collaboration partners in the USA (Pennsylvania State University).

\section{Methods and analysis}

The project PELI-D is designed as a prospective observational study with 3-year project duration from 2017 to 2020 (figure 1). The project consists of two consecutive phases. Both project phases will take place in the following care settings: nursing homes, home care and adult daycares.

Within the first phase 'translation', a culture-sensitive translation of the PELI was conducted (finished in October 2018). Goals were to (a) systematically translate the original PELI version for nursing homes from English into German in a culture-sensitive manner, (b) validate the questionnaire linguistically, (c) discuss the translated PELI with professional caregivers, professional translators and the developer and $(\mathrm{d})$ provide a modified version of the PELI-D for each of the focused care settings for the phase two.

Afterwards, sessions for job training (3 days training for professional caregivers) and subsequent training on the job, marked by close communication, support and feedback while conducting the first PELI-D questionnaires in the care settings, were realised by the end of January 2019. Additional training sessions will be provided if needed (eg, fluctuation of staff, participation of a care facility).

Phase two of our 'pilot study' started in January 2019. One aim of this phase is to analyse whether settingspecific versions are reliable, feasible and practical for identifying preferences of older people in need of care in German care settings. Primarily, a test-retest reliability indicator of the instrument at baseline (T0) and at 2-week follow-up (T1) will be conducted with the group of older people in need of care. Additionally, we will test the proxy version of the PELI-D with their close relatives in close contact. Another aim of the pilot study is the analysis of the stability of preferences. Thus, a 3-month follow-up (T2) will be conducted with older people in need of care. Furthermore, questions regarding satisfaction in fulfilling the preferences and reasons for personal or institutional barriers that hinder adherence to the preferences will be asked (T2). To determine the level of understanding and meaning of the setting-specific PELI-D, a few older people in need of care (T1) and their close relatives (T0) will participate in a cognitive interview ${ }^{12}$ in each care setting.

Each of our practice partners defined key persons who will coordinate the project within their care facilities. 


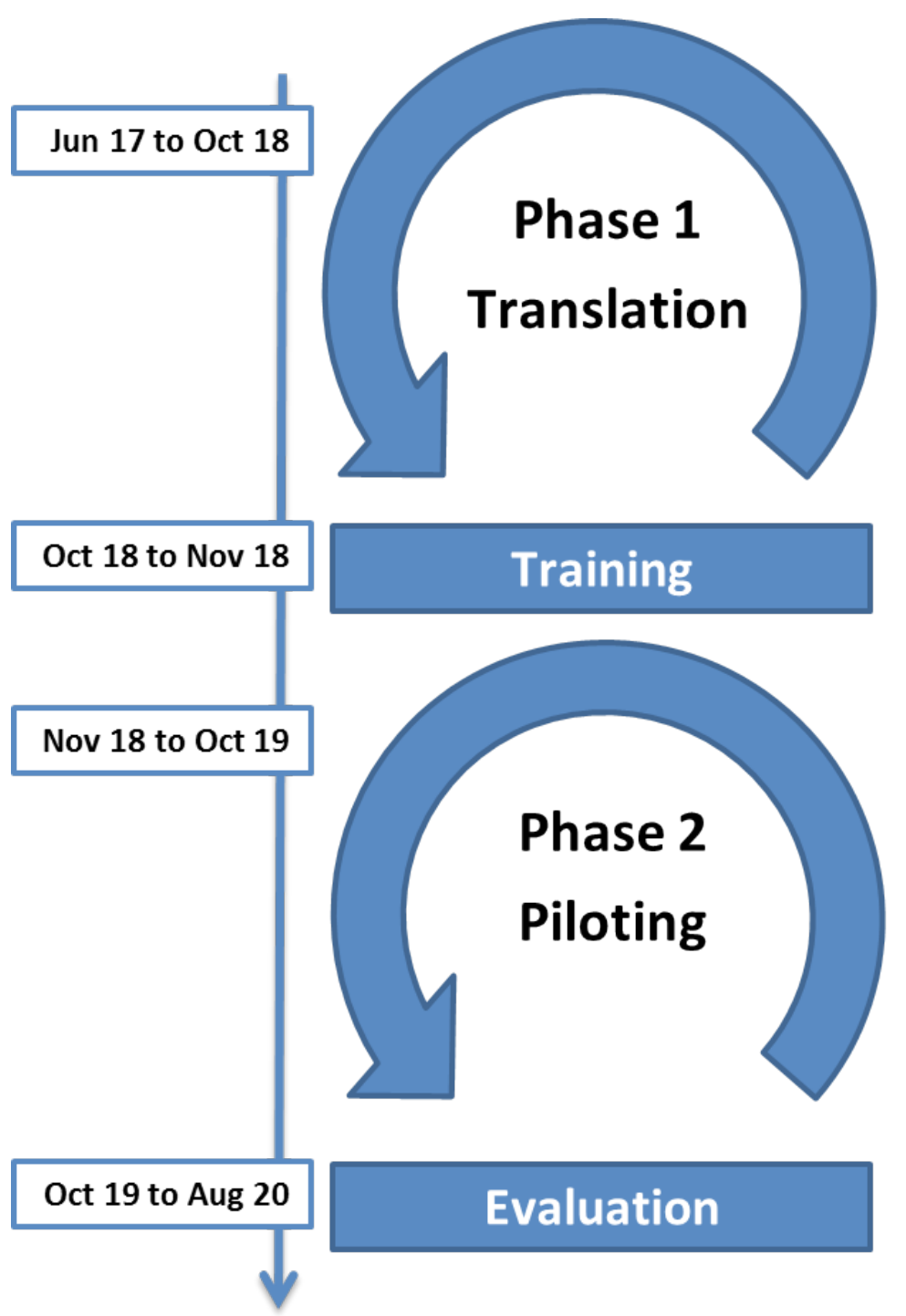

\section{Central aspects}

I. Culture-sensitive translation of the original PELI questionnaire for nursing homes.

Modification of the translated PELI-D
versions.

II. Training of the professional caregivers in usage of the PELI-D versions.
III. Psychometric testing of the PELI-D versions.

Reflection of the feasebility and practicability of the PELI-D versions.

Linguistic validation of the PELI-D versions by elderly persons in need of care and their close relatives.

\section{Analysis and discussion with the developer.}

Figure 1 Phases and schedule of the project PELI-D. PELI-D, preferences for everyday living inventory.

Eleven care facilities will be involved in the pilot study, including three nursing homes, three home care services and five adult daycare services.

Patients and public were not actively involved in the research project and in research organisation.

\section{PHASE 1: CULTURE-SENSITIVE AND CONTENT-RELATED TRANSLATION}

Participants, setting and recruitment

The German PELI-D project team consists of one project leader, three research associates and two research assistants. Kimberly Van Haitsma and her team from The Pennsylvania State University will collaborate with the German project team over the whole project period, specifically during the backward translation. The two forward translators are researchers at the DZNE (site Witten); both are native German speakers with high-level English skills, theoretical knowledge about professional care and experience in the field of nursing care. The two back translators are professional translators; both are native English speakers with excellent German language skills and experience in healthcare research. The expert panel discussion includes researchers and students of the DZNE (site Witten) who have specific scientific knowledge of care and experience in the field of acute and long-term care.

The 11 professional caregivers who were involved in the cognitive debriefing ${ }^{13}$ are nurses at the participating care facilities, have at least 4 years of experience in professional care (including a 3-year duration vocational training) and have been an employee for more than 6 months at one of the participating care facilities (nursing home, home care and adult daycare).

\section{The translation process}

A culture-sensitive and content-related translation for the care settings was conducted with the current item-based PELI questionnaire for nursing homes (long version). ${ }^{7}$ Among other specific methods for translation, ${ }^{14}$ the recommendations of the Task Force on Translation and Cultural Adaptation of the International Society for 
Pharmacoeconomics and Outcome Research (ISPOR) were followed. ${ }^{13}$ To account for the conditions of the different care settings, the perspectives of professional caregivers were included within the proposed procedure of the cognitive debriefing. The ISPOR specification was extended by a recommended expert panel discussion. $^{16}{ }^{17}$ While conducting the ISPOR recommendations, we continuously evaluate the cultural adaption of the PELI instrument. Separate articles will address this evaluation.

Step I: The latest PELI version was designed and tested for nursing homes (PELI $\mathrm{Original}_{\text {al }}$ ). According to the ISPOR recommendations, ${ }^{13}$ two forward translations were conducted (PELI-D ${ }_{\mathrm{FT} 1}$ and PELI-D ${ }_{\mathrm{FT} 2}$ ) first, linguis-

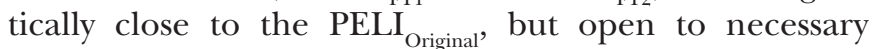
culture-specific and setting-specific modifications. As a result, a preliminary forward translated version of the PELI-D (PELI-D FT3 $_{\text {) }}$ was drafted. Next, a modified draft was created (PELI-D $\left.{ }_{E P 1}\right)$ based on the discussion of the PELI-D ${ }_{\mathrm{FT} 3}$ with an expert panel. ${ }^{17}$ Each item was discussed according to the following criteria until a qualitativebased consensus was reached: (a) Does the translation corresponds to the meaning of the original text? (b) Is the translated content relevant (eg, response options) for the setting? (c) Is the translation understandable for caregivers and older people in need of care? Furthermore, we organised a 3-day workshop with the PELI developer to clarify culture-sensitive care service semantics and culture-specific contents. The PELI-D ${ }_{\mathrm{EP} 1}$ was reviewed and modified to produce the PELI-D ${ }_{\mathrm{EP} 2}$ draft.

Step II: During the subsequent cognitive debriefing, ${ }^{13}$ the linguistic validation, suitability and understandability of the PELI-D ${ }_{\mathrm{EP} 2}$ was evaluated by conducting settingspecific cognitive interviews ${ }^{12}$ with all 11 professional caregivers from the three different care settings. The results of the cognitive interviews were intensively discussed with regard to aspects of culturally sensitive language and a setting-specific perspective. Both the language of the PELI- $D_{\mathrm{EP} 2}$ and the relevance of the items for the care settings were discussed by the expert panel (site Witten). Therefore, the content validity index, ${ }^{18} 19$ a descriptive statistical method for reducing the amount of items, was used as an additional tool. The item reduction was based on the professional caregivers' estimation of the suitability and understandability of the translated PELI items. As a result, three setting-specific German draft versions with a reduced number of items for nursing homes (PELI-D ${ }_{\mathrm{NH} 1}$ ), home care $\left(\right.$ PELI-D $\left.\mathrm{HCl}_{\mathrm{HC}}\right)$ and adult daycares $\left(\right.$ PELI-D $\left.\mathrm{ADCl}_{\mathrm{ADC}}\right)$ were generated. Those differentiations seemed necessary since not every item was suitable and understandable in the same way for every setting from a practical and scientific point of view. Parallel to the cognitive debriefing, PELI $\mathrm{FP}_{\mathrm{EP}}$ was independently back translated by two external translators (PELI-D ${ }_{\mathrm{BT} 1}$ and PELI-D $\mathrm{BT}_{2}$ ).

Step III: In the last step, the two back translated drafts PELI-D ${ }_{\mathrm{BT1}}$ and PELI-D ${ }_{\mathrm{BT} 2}$, PELI-D ${ }_{\mathrm{NH} 1}$, PELI-D ${ }_{\mathrm{HCl}}$, PELI-D ${ }_{\mathrm{ADCl}}$ and PELI ${ }_{\text {Oriminal }}$, were discussed within the project team, with the PELI developer and the backward
Table 1 Milestones of the culture-sensitive translation and modification of the PELI-D versions

\begin{tabular}{|c|c|c|}
\hline Step & $\begin{array}{l}\text { Initial situation / } \\
\text { requirement }\end{array}$ & Results \\
\hline I & $\begin{array}{l}\text { Original PELI for } \\
\text { nursing homes } \\
\text { (PELI Original) }\end{array}$ & $\begin{array}{l}\text { Preliminary PELI-D version } \\
\left(\text { PELI-D }_{\text {EP2 }}\right)\end{array}$ \\
\hline \multirow[t]{2}{*}{ II } & $\begin{array}{l}\text { Cognitive debriefing } \\
\text { with PELI-D (PELI- } \\
\left.D_{E P 2}\right)\end{array}$ & $\begin{array}{l}\text { Setting-specific PELI-D versions } \\
\left(\text { PELI-D }{ }_{\mathrm{NH} 1, \mathrm{HC} 1, \mathrm{ADC} 1}\right)\end{array}$ \\
\hline & $\begin{array}{l}\text { Back translation of } \\
\left.\text { PELI-D (PELI-D } \text { EP2 }_{1}\right)\end{array}$ & $\begin{array}{l}\text { Back translated PELI-D versions } \\
\left(\text { PELI- } \mathrm{D}_{\mathrm{BT} 1, \mathrm{BT} 2}\right)\end{array}$ \\
\hline III & $\begin{array}{l}\text { Discussion of the } \\
\text { results from step II }\end{array}$ & $\begin{array}{l}\text { Reviewed setting-specific } \\
\text { PELI-D versions: } \\
\text { Global version (PELI-D }{ }_{\text {EP3 }} \text { ) } \\
\text { including } 72 \text { items } \\
\text { Nursing home version (PELI- } \\
D_{\mathrm{NH} 2} \text { ) including } 65 \text { items } \\
\text { Home care version (PELI- } \\
D_{\mathrm{HC} 2} \text { ) including } 55 \text { items } \\
\text { Adult daycare version (PELI- } \\
D_{\mathrm{ADC} 2} \text { ) including } 54 \text { items }\end{array}$ \\
\hline
\end{tabular}

PELI-D, preferences for everyday living inventory.

translators. Following this discussion, updated settingspecific German versions (PELI-D ${ }_{\mathrm{NH} 2}$, PELI-D $\mathrm{HC}_{\mathrm{HC}}$ and PELI-D ${ }_{\mathrm{ADC2}}$ ) were defined. The global version $\left(\mathrm{PELI}_{\mathrm{H}} \mathrm{D}_{\mathrm{EP} 3}\right)$ with all 72 items was not considered for the settingsspecific testing in phase two. The modified setting-specific German versions are similar in their structure (and to the PELI $\left._{\text {Original }}\right)$ and have been linguistically validated, but they are different in the number of preferences (table 1 ).

\section{PHASE 2: PILOTING THE PELI-D}

Before the start of the second study phase, professional caregivers were successfully trained in using the PELI-D questionnaire. In the second study phase, the focus is on the reliability, feasibility and practicability of settingspecific PELI-D versions (PELI-D $\mathrm{NH}_{2}$, PELI-D $\mathrm{HC}_{\mathrm{H} 2}$ and PELI$\mathrm{D}_{\mathrm{ADC2}}$ ). These aspects are being validated over a 10-month period between January and October 2019.

\section{Training of staff}

Since professional caregivers are assessing the preferences of older people in need of care, we provided setting-specific training. The first assessment training took place in October/November 2018 in the facilities of our practice partners. Over 3 days, professional caregivers learnt how to apply the setting-specific PELI-D versions, which instruments are necessary and how to apply them (eg, MMSE), and how to determine what additional data need assessing and when to collect preferences within the nursing process. They were also trained how to conduct the interviews regarding questions of satisfaction and barriers in addition to the individual preferences. Further follow-up training sessions were and will be conducted in 


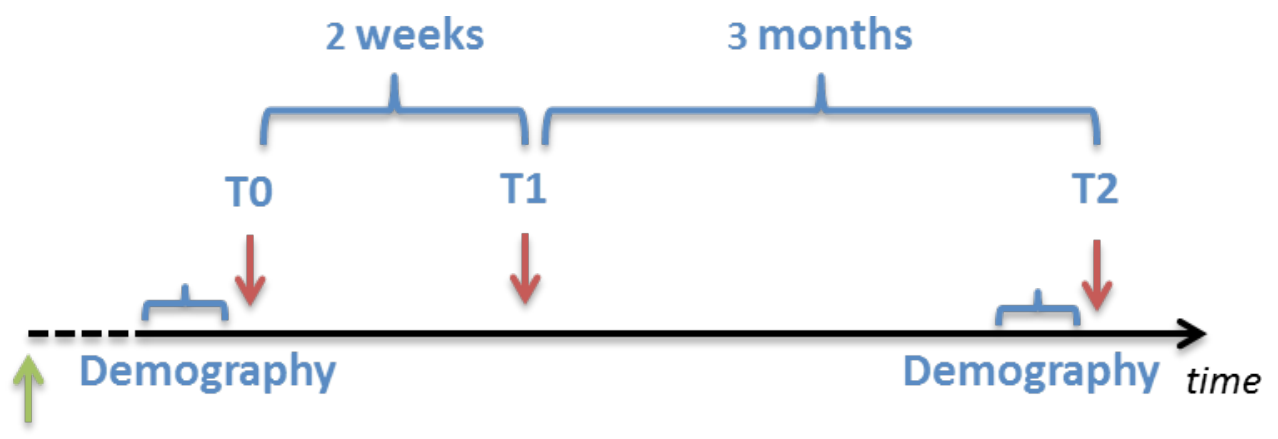

\section{Start of the utilization of the care service}

Figure 2 Schedule for the project PELI-D for one person within the pilot study (phase two). PELI-D, preferences for everyday living inventory.

2019. To date, a total of 38 professional caregivers were successfully trained for the use of the PELI-D.

The second assessment training was carried out from November 2018 to January 2019 to facilitate the application of the PELI-D at the care facilities. Members of the PELI-D project team visited the participating care facilities to identify questions and problems while using the PELI-D and to clarify content-specific or infrastructurerelated issues.

\section{Data collection}

Preferences of older people in need of care will be assessed from January to October 2019 by using the setting-specific German PELI versions administered by trained professional caregivers. To examine reliability, the PELI-D data of 75 older people in need of care in each of the three different care settings will be collected at three time points (T0: baseline, T1: 2 weeks after baseline and T2: 3-month follow-up) (figure 2). Furthermore, within the 3-month follow-up (T2), older people in need of care will be asked questions regarding satisfaction and barriers as well as their personal preferences. The described data collection will be embedded in the nursing process.

To calculate inter-rater reliability, a proxy-version of the PELI-D questionnaire will additionally be conducted for close relatives, independently of their older people in need of care. The close relatives will complete the proxy version of the PELI-D questionnaire on their own.

Some of the participants in need of care and their close relatives will separately take part in a cognitive interview. ${ }^{12}$ The aim is to learn more about their understanding of the meaning of the PELI-D items. The involved professional caregivers will be asked about the feasibility and practicability of the PELI-D in focus group interviews. Two focus group interviews will be conducted (March and September 2019).

\section{Participants, inclusion criteria and recruitment}

To realise a required sample size in order to obtain a valid measurement accuracy for calculating the reliability, considerations were made with regard to the resources available in the project. At least 75 older people in need of care will be recruited consecutively ${ }^{20}$ within the different care settings: nursing homes, home care and adult daycares. The sample size estimation for the measurements of test-retest reliability and possible impact of fixed numbers of participant are described in the next section.

Inclusion and exclusion criteria have been established. The current state of research indicates that reliable answers to the PELI questions require some level of cognitive ability. ${ }^{8}$ For this purpose, the cognitive status will be assessed with the DZNE-licensed version of the MMSE $^{21}$ (excluding a cut-off value $\leq 19$ ), so that only older people with no or mild cognitive impairments (mild types of dementia) are included. ${ }^{22}$ For ethical reasons, older people who receive palliative medical care or have a poor or critical health condition will be excluded from this study. Furthermore, PELI-D-trained professional caregivers will evaluate the patient's general health condition with the EQ-5D-5L, ${ }^{23}$ anxiety symptoms with one specific question from the $\mathrm{PHQ}^{24}$ and depression syndrome with the PHQ-2. ${ }^{25}$ Additionally, pain (the quality and grade of the pain) can be obtained from nursing documentation. These aspects provide a picture of the general health condition and can be used as exclusion criteria by trained professional caregivers.

The recruitment of older people in need of care will be conducted by the PELI-D-trained professional caregivers of our practice partners (wider area of Dusseldorf and Cologne). Each older person in need of care should be included immediately after the care service starts; further inclusion criteria are checked when the older person is interviewed using the PELI-D questionnaire.

Furthermore, 75 close relatives of the recruited older people per setting will be asked by the PELI-D-trained professional caregivers to participate in the study and to complete a proxy version of the PELI-D. An agreement from the older people in need of care is a prerequisite before recruitment of the proxy. If the close relative is not able or willing to participate, a professional caregiver of the care facility, who knows the person in need of care best-will be selected by a PELI-D-trained professional caregiver, may complete the proxy-version of the PELI-D. A requirement for the proxy survey is that this professional 


\begin{tabular}{llll}
\multicolumn{4}{l}{ Table 2} \\
\multicolumn{4}{l}{ Lower bounds of a $95 \% \mathrm{Cl}$ for two ratings on 75} \\
\hline Estimate & ICC & Kappa & Total agreement \\
\hline $\mathbf{0 . 5 0}$ & 0.33 & 0.23 & 0.40 \\
$\mathbf{0 . 5 5}$ & 0.39 & 0.28 & 0.43 \\
$\mathbf{0 . 6 0}$ & 0.45 & 0.33 & 0.49 \\
$\mathbf{0 . 6 5}$ & 0.52 & 0.38 & 0.54 \\
$\mathbf{0 . 7 0}$ & 0.58 & 0.43 & 0.60 \\
$\mathbf{0 . 7 5}$ & 0.65 & 0.48 & 0.64 \\
$\mathbf{0 . 8 0}$ & 0.72 & 0.54 & 0.70 \\
$\mathbf{0 . 8 5}$ & 0.79 & 0.60 & 0.76 \\
$\mathbf{0 . 9 0}$ & 0.86 & 0.67 & 0.82 \\
\hline
\end{tabular}

ICC, intraclass correlation coefficient.

caregiver has not conducted the PELI-D interview with the person in need of care; otherwise, they would already know the preferences of the older people in need of care that will be asked with the PELI-D instrument.

\section{Reliability: sample size estimation and statistical analysis}

Two kinds of reliability will be assessed: test-retest reliability at different measurement points for the self-rating of older people in need of care and inter-rater reliability of self-rating and proxy-rating at the same time point. Three statistics, Cohen's kappa,${ }^{26}$ intraclass correlation coefficients (ICC) ${ }^{27}$ and total agreement, ${ }^{28}$ will be used to assess the two reliability indicators of the preference importance ratings of the PELI-D questionnaire and the summary scores thereof. These statistics were chosen according to the guidelines by Kottner et al (table 2 in article) ${ }^{29}$ and Koo and Li (figure 1 in article). ${ }^{30}$

As reported above, sample size was fixed at 75 older people in need of care for each care setting, for example, expected new admissions to the care provider over the 1-year duration. The sample size of the close relatives of the older people in need of care follows this sample size calculation. Therefore, on the basis of $95 \%$ CIs for the ICC, kappa statistic and total agreement, 75 participants were estimated according to Bonett, ${ }^{31}$ Donner and Rotondi ${ }^{32}$ and Agresti and Coull, ${ }^{33}$ respectively, with software $\mathrm{R}^{34}$ (table 2). Cut-off values that define ranges of 'poor', 'moderate' and 'good' reliability are available in the literature ${ }^{526}{ }^{30}$ With at least $\mathrm{n}=75$ samples, CIs will be low enough to fit within one of these ranges for ICC and total agreement; however, the kappa statistic may have a wider CI, spanning more than two ranges depending on the value of kappa (table 2). The reliability measurement, which was carried out on the basis of these estimated values, can lead to informative statements about the PELI-D. With 60 participants, CIs increase and kappa estimates become uninformative, although ICC and total agreement are still useable. With 45 participants, all three statistics have such wide CIs that their estimates are uninformative.

\section{Cognitive interviews}

Between January and October 2019, five to 10 (plus their close relatives) out of the 75 recruited older people in need of care per setting will be asked to take part in the cognitive interview. The older people will be asked if they are interested in doing an interview within the next few days after they finish the PELI-D for the second time (T1). Their close relatives will be asked if they are interested in an interview after they finish the proxy version of the PELI-D. A member of the PELI-D team will conduct the interviews with the older people in need of care and with their close relatives separately. The interview will be either a face-to-face interview or a phone interview. The cognitive interviews will be conducted for each care setting and are similar to those in project phase one. In contrast to the cognitive interview in phase one, fewer questions will be asked, the language will be simplified and an open introductory question will be asked. The objective is to ask about the meaning of and the ability to understand the setting specific preference-based items of the reduced PELI-D questionnaires. These interviews will be analysed using a modified qualitative structuring content analysis. ${ }^{35} 36$

\section{Focus group interviews}

The primary focus of the project is to analyse the feasibility and applicability of the German setting-specific PELI-D versions in practice. All PELI-D-trained professional caregivers will be asked to take part in two focus group interviews (March and September 2019). Focus group interviews collect information provided by a relatively heterogeneous group's discussion beyond the focused objective that is generated by the researcher. ${ }^{37}$

In March 2019, the first focus group interview will be conducted in each setting with a focus on practicability and feasibility of the setting-specific PELI-D version. In September 2019, the second focus group interview including all three settings will focus on aspects of the applicability and feasibility of the PELI-D in the nursing process, gathering perspectives from within and across care settings. Approximately five to eight PELI-D-trained professional caregivers from all three care settings will be asked to participate in this second focus group interview.

To analyse these data, a modified qualitative structuring content analysis will be used. ${ }^{35}{ }^{36}$ It is expected that this analysis will be further supplemented by variants of the structuring qualitative content analysis, which will be systematically merged; we will refer to Schreier's suggestion of a modular structure for this purpose. ${ }^{36}$

\section{DISSEMINATION}

All personal information will be deidentified with the identification code kept in a secured location apart from the rest of the study data. Only qualified and study-related personnel will be allowed to access the data. The results of the study will be distributed widely through peer-reviewed journals, conferences and international meetings. 
An integrated knowledge translation approach is used in this study. Collaborations with multiple sites internationally will hopefully result in increased use of the PELI (part or in whole) in the future.

One goal of our pilot study is to make the PELI-D available for different care settings free of charge. Furthermore, a dialogue regarding the embedding of the PELI-D (or content-related elements of the questionnaire) in care documentation should be stimulated within the care settings. In addition, the results of this pilot study will help to further develop the original PELI questionnaire and generate additional preference-based items in a national and international context.

There is no intention to compare different care facilities, older people in need of care or the results of our practice partners with respect to their outcomes. The results of the PELI-D questionnaires of the older people in need of care will be sent directly, via a special outcome sheet, to the participating facility for their own use, respecting data-protection requirements. Finally, the results of the study will be primarily published in open access journals. All data files (eg, data sets and interview recordings) are stored on a server of the DZNE. A publication of the data files is not planned. Study team members will present the results at international and national conferences.

\section{Author affiliations}

${ }^{1}$ German Center for Neurodegenerative Diseases (DZNE) Site Witten, Witten,

Germany

${ }^{2}$ Department of Nursing Science, University of Witten/Herdecke, Faculty of Health, Witten, Germany

${ }^{3}$ Write-English, Cologne, Germany

${ }^{4}$ White-English Services, Zurich, Switzerland

${ }^{5}$ Diocesan Caritas Association for the Archdiocese of Cologne, Cologne, Germany

${ }^{6}$ Diaconia, Dusseldorf, Germany

${ }^{7}$ Polisher Research Institute, Penn State College of Nursing, University Park,

Pennsylvania, USA

Acknowledgements The authors acknowledge the cooperation partners Diaconia and Caritas in Germany and the Polisher Research Institute in the US in various phases of the study.

Contributors RMa, PD, STI, BJM and SAM conceptualised the study. STI and BJM prepared the initial draft of the manuscript equally. RMa supervises all steps of the project. PD coordinates the project. STI is responsible for the conception of phase one and two and the training of the professional caregivers. STI circulated the manuscript among the authors. BJM is responsible for the statistical methods and instruments across phase two and for the training of the MMSE. SAM conceptualised the statistical methods for the reliability analyses and executed the sample size calculations. NAE translated the PELI into German and was part of the expert panel. VHK contributes to the overall methodology of the pilot study and the translation process. $\mathrm{KH}$ and $\mathrm{RN}$ provide care facilities, organise the trainings and support the pilot study in their care facilities (eg, recruitment). MC and RMi assist the project team. MWR is responsible for the technical realisation (eg, data collection) and data management. WJ and WA conducted the back translation. All authors read and approved the final manuscript. All authors worked along the four main criteria based on the ICMJE recommendations.

Funding This study is nine-tenths funded by the Stiftung Wohlfahrtspflege Nordrhein-Westfalen (Foundation for Welfare Work North Rhine-Westphalia) (funding number: SW-620-6734-Z), a foundation that works for the sustainable improvement of people's living conditions. The remaining tenth is funded by the German Center for Neurodegenerative Diseases (registered association), which is part of the Helmholtz-Gemeinschaft (Helmholtz Association); its task is to pursue the long-term research objectives of the state and society. Both funding bodies advocate open access research publication.
Competing interests None declared.

Patient consent for publication Not required.

Ethics approval This study has been approved by the internal quality control committee of the German Center for Neurodegenerative Diseases (site Bonn) within the Helmholtz Association (ID number: WI029 PELI-D) and the ethics committee of the German society of nursing science (registered association) (ID number: 18-010).

Provenance and peer review Not commissioned; externally peer reviewed.

Open access This is an open access article distributed in accordance with the Creative Commons Attribution Non Commercial (CC BY-NC 4.0) license, which permits others to distribute, remix, adapt, build upon this work non-commercially, and license their derivative works on different terms, provided the original work is properly cited, appropriate credit is given, any changes made indicated, and the use is non-commercial. See: http://creativecommons.org/licenses/by-nc/4.0/.

ORCID iD

Tobias Ingo Stacke http://orcid.org/0000-0002-3556-8128

\section{REFERENCES}

1 Bundesministerium des Innern, Jedes Alter zählt. Demografiestrategie der Bundesregierung. In: Bundesministerium des Innern. Berlin, 2012

2 Bundesministerium für Arbeit und Soziales. Unser Weg in eine inklusive Gesellschaft - Der Nationale Aktionsplan der Bundesregierung zur Umsetzung der UNBehindertenrechtskonvention. In: Bundesministerium für Arbeit und Soziales. Berlin, 2011.

3 Bundesministerium für Arbeit und Soziales. Entwurf eines Gesetzes zur Stärkung der Teilhabe und Selbstbestimmung von Menschen mit Behinderungen (Bundesteilhabegesetz - BTHG). Berlin, 2016.

4 Roes M, Bieber A, Burbaum J, et al. Der Expertenstandard Beziehungsgestaltung in Der Pflege von Menschen MIT Demenz. Osnabrück: Deutsches Netzwerk für Qualitätsentwicklung in der Pflege, 2018: 24-69.

5 Van Haitsma K, Abbott KM, Heid AR, et al. The consistency of selfreported preferences for everyday living: implications for personcentered care delivery. J Gerontol Nurs 2014;40:34-46.

6 Carpenter BD, Van Haitsma K, Ruckdeschel K, et al. The psychosocial preferences of older adults: a pilot examination of content and structure. Gerontologist 2000;40:335-48.

7 Van Haitsma KS. Preferences for Everyday Living Inventory - Nursing Home version (PELI-NHC), 2013.

8 Van Haitsma KS, Kleban M, Curyto KJ, et al. Assessing preferences for everyday living in the nursing home: reliability and concordance issues 2014.

9 Van Haitsma K, Abbott KM, Arbogast A, et al. A Preference-Based model of care: an integrative theoretical model of the role of preferences in Person-Centered care. Gerontologist 2019;30:gnz075.

10 Van Haitsma K, Crespy S, Humes S, et al. New toolkit to measure quality of person-centered care: development and pilot evaluation with nursing home communities. J Am Med Dir Assoc 2014;15:671-80.

11 Rommerskirch M, Purwins D, Stacke TI, et al. The recording of preferences for everyday living of people with care needs in different nursing settings in Germany. An explorative analysis of nursing records. Artikel in Vorbereitung 2019.

12 Willis GB. Cognitive interviewing: a tool for improving questionnaire design. Thousand Oaks, Calif, London: Sage, 2005.

13 Wild D, Grove A, Martin M, et al. Principles of good practice for the translation and cultural adaptation process for patient-reported outcomes (pro) measures: report of the ISPOR Task force for translation and cultural adaptation. Value Health 2005;8:94-104.

14 Acquadro C, Conway K, Hareendran A, et al. Literature review of methods to translate health-related quality of life questionnaires for use in multinational clinical trials. Value Health 2008;11:509-21.

15 Maneesriwongul W, Dixon JK. Instrument translation process: a methods review. J Adv Nurs 2004;48:175-86.

16 Hoben M, Bär M, Mahler C, et al. Linguistic validation of the Alberta context tool and two measures of research use, for German residential long term care. BMC Res Notes 2014;7:1-12.

17 Hoben M, Mahler C, Bär M, et al. German translation of the Alberta context tool and two measures of research use: methods, challenges and lessons learned. BMC Health Serv Res 2013;13:1-12.

18 Polit DF, Beck CT. The content validity index: are you sure you know what's being reported? critique and recommendations. Res Nurs Health 2006;29:489-97. 
19 Polit DF, Beck CT, Owen SV. Is the CVI an acceptable indicator of content validity? Appraisal and recommendations. Res Nurs Health 2007;30:459-67.

20 Suresh S. Nursing research and statistics. London: Elsevier Health Sciences, 2014.

21 Folstein MF, Folstein SE, McHugh PR. "Mini-mental state". J Psychiatr Res 1975;12:189-98.

22 DGPPN. Deutsche Gesellschaft für Psychiatrie und Psychotherapie PuNDaDGfND. S3-Leitlinie "Demenzen". 106. Bonn, 2016.

23 Herdman M, Gudex C, Lloyd A, et al. Development and preliminary testing of the new five-level version of EQ-5D (EQ-5D-5L). Qual Life Res 2011;20:1727-36.

24 Spitzer RL, Kroenke K, Williams JB. Validation and utility of a selfreport version of PRIME-MD: the PHQ primary care study. primary care evaluation of mental disorders. patient health questionnaire. JAMA 1999;282:1737-44.

25 Kroenke K, Spitzer RL, Williams JBW. The patient health Questionnaire-2: validity of a two-item depression screener. Med Care 2003;41:1284-92.

26 Landis JR, Koch GG. The measurement of observer agreement for categorical data. Biometrics 1977;33:159-59.

27 Zou G, Donner A. Confidence interval estimation of the intraclass correlation coefficient for binary outcome data. Biometrics 2004;60:807-11.
28 Kottner J, Dassen T. Interpreting interrater reliability coefficients of the Braden scale: a discussion paper. Int J Nurs Stud 2008;45:1238-46.

29 Kottner J, Gajewski BJ, Streiner DL. Guidelines for reporting reliability and agreement studies (GRRAS). Int J Nurs Stud 2011;48:659-60.

30 Koo TK, Li MY. A guideline of selecting and reporting intraclass correlation coefficients for reliability research. J Chiropr Med 2016;15:155-63.

31 Bonett DG. Sample size requirements for estimating intraclass correlations with desired precision. Stat Med 2002;21:1331-5.

32 Donner A, Rotondi MA. Sample size requirements for interval estimation of the kappa statistic for interobserver agreement studies with a binary outcome and multiple raters. Int J Biostat 2010;6.

33 Agresti A, Coull BA. Approximate is Better than "Exact" for Interval Estimation of Binomial Proportions. Am Stat 1998;52:119-26.

34 R Core Team. R: a language and environment for statistical computing. R Foundation for Statistical Computing, 2017.

35 Schreier M. Qualitative content analysis in practice. London: Sage, 2012.

36 Schreier M. Varianten qualitativer Inhaltsanalyse: ein Wegweiser Im Dickicht Der Begrifflichkeiten. Forum Qualitative Sozialforschung 2014;15.

37 Schulz M, Mack B, Renn O. Fokusgruppen in Der empirischen Sozialwissenschaft. von der Konzeption bis Zur Auswertung. Stuttgart: VS Verlag für Sozialwissenschaften, 2012. 